\title{
Una experiencia para fortalecer los procesos de enseñanza de la programación mediante el uso de entornos virtuales de aprendizaje
}

\author{
Aguirre, Jesús Francisco, Viano, Hugo josé, García, Berta \\ Departamento de Informática - Fac. de Cs. Físico Matemáticas y Naturales \\ Universidad Nacional de San Luis - San Luis, Argentina \\ \{jaguirre@unsl.edu.ar\}\{hviano, bertae.garcia\}@gmail.com
}

\begin{abstract}
Resumen. Este trabajo describe y presenta los primeros resultados de una experiencia desarrollada en el contexto de la asignatura Programación, de la carrera Ingeniería Electrónica con Orientación en Sistemas Digitales. A partir de un diagnóstico inicial se identificaron algunas problemáticas comunes a los estudiantes tales como: falta de hábitos de estudio, escaso nivel de motivación y dificultad para cumplir con los horarios establecidos. Con el propósito de lograr mayor participación y proveer acceso permanente a los materiales de estudio se creó un aula virtual. Se propusieron actividades colaborativas y se diagramó un práctico de máquina en forma grupal para resolver en un entorno de desarrollo integrado de software libre.

Puede concluirse que los recursos y estrategias utilizadas impactaron positivamente en los procesos de aprendizaje, favorecieron la colaboración y tutorización, proporcionaron información valiosa para el seguimiento de los estudiantes y permitieron aprender más allá de los límites del aula presencial.
\end{abstract}

Palabras Clave: entornos de aprendizaje virtual, software libre, entornos de desarrollo integrado, aprendizaje colaborativo.

\begin{abstract}
This work describes and presents the first results of an experience conducted in the context of the subject Programming, which is part of the academic program Electronic Engineering with an Orientation to Digital Systems. An initial diagnosis allowed for the identification of some problems typical of students, such as: lack of study habits, poor motivation level, and difficulty to meet scheduled times. A virtual classroom was created with the purpose of achieving greater participation on the part of students and providing permanent access to study materials. Collaborative activities were put forward, and a practical group work was planned to be solved in an integrated development environment of free software. Results suggest that the resources and strategies used had a positive impact on the learning processes, favored collaboration and mentoring, provided useful information for student follow-up, and allowed for learning beyond the limits of a conventional classroom.
\end{abstract}

Keywords: virtual learning environments, free software, integrated development environments, collaborative learning 


\section{Introducción}

El impacto generado en nuestra sociedad debido a una evolución constante de la informática, permitió la aparición de nuevas Tecnologías de la Información y las Comunicaciones a las que comúnmente se las denomina TIC. El surgimiento del movimiento de Software Libre (SL) propone un nuevo modelo de trabajo sobre las libertades de los usuarios y la propiedad intelectual del software desarrollado. En la actualidad, ha demostrado tener viabilidad tanto técnica como económica y las estadísticas reflejan un crecimiento sostenido de su uso.

Del análisis realizado por la cátedra sobre los resultados obtenidos en los últimos años de la asignatura "Programación", se identificaron diferentes factores que influyeron en forma negativa en los aprendizajes. A partir de este diagnóstico, se propusieron estrategias para promover el compromiso, la motivación y el interés de los alumnos.

La elección de un Entorno Virtual de Aprendizaje (EVA), y en este caso particular del entorno "Aulas virtuales" implementado sobre la plataforma Moodle [1], responde a la necesidad de incrementar la participación de los alumnos que plantean conflictos para realizar las actividades presenciales. El aula virtual creada para la asignatura aporta un espacio de participación flexible como complemento al aula presencial. $[2,3]$ El uso de un entorno de desarrollo integrado para resolver grupalmente un problema computacional responde a la necesidad de promover aprendizajes significativos a partir de la interacción con otros estudiantes.

Este trabajo relata una experiencia desarrollada en el marco de la enseñanza de la programación a alumnos de segundo año de la carrera de Ingeniería Electrónica con orientación en Sistemas Digitales, usando herramientas de SL y un aula en un EVA. El mismo se organiza de la siguiente manera: se sintetiza el marco teórico y el contexto del trabajo; luego se describen el diagnóstico inicial y las estrategias propuestas; posteriormente se explica la forma de recolección de los datos que permitieron evaluar la propuesta, obtener conclusiones y delinear futuras líneas de trabajo.

\section{Marco teórico}

La aparición de nuevas formas de organización económica, social, política y cultural trae consigo nuevas maneras de trabajar, comunicarnos, aprender, pensar y vivir. A este nuevo orden social se lo llama Sociedad de la Información [4]. Las TIC ofrecen la posibilidad de reconstruir y reinterpretar las posibilidades de enseñar, de acuerdo con el marco socio-educativo-cultural de referencia.

El desafío para la educación superior consiste en encontrar modelos más abiertos y flexibles, donde el estudiante pueda hacerse responsable de la construcción de su propio aprendizaje. Para ello, la enseñanza debe adoptar metodologías más activas. En este sentido, el uso de entornos virtuales amplía las posibilidades de aprendizaje personalizado y seguimiento de los alumnos por parte de los equipos de cátedra.

Diseñar un espacio virtual como apoyo a la clase presencial promueve la construcción de conocimiento manteniendo cierta independencia del espacio y el 
tiempo pedagógicos. En estas propuestas el docente asume un rol de guía, centrado en el proceso más que en los resultados, organizando y secuenciando el material didáctico de acuerdo a las características de los estudiantes destinatarios, el contexto en el que se produce el proceso, la organización de la información y la tutorización.

Promover aprendizajes significativos en los estudiantes implica una participación más activa de quienes aprenden y da lugar al aprendizaje colaborativo.

El aprendizaje colaborativo es definido por Johnson [5] como: "el conjunto de métodos de instrucción o entrenamiento para su uso en grupos, así como de estrategias para propiciar el desarrollo de habilidades mixtas: aprendizaje y desarrollo personal y social. El aprendizaje colaborativo virtual también es entendido como un proceso de construcción social de conocimiento. A partir del trabajo conjunto y las metas comunes, se da una "reciprocidad entre un conjunto de individuos que saben diferenciar y contrastar sus puntos de vista de tal manera que llegan a generar un proceso de construcción de conocimiento. Es un proceso en el que cada individuo aprende más de lo que aprendería por sí solo, fruto de la interacción de los integrantes del equipo" [6]. En el desarrollo de un grupo, por tanto, la interacción se convierte en un elemento clave, teniendo en cuenta que es el proceso esencial de reunir las contribuciones de los participantes en la co-creación de conocimiento [7].

Si bien el uso de tecnologías no implica nuevas formas de aprendizaje, la presencia de computadoras, equipos portátiles, teléfonos inteligentes, instrumentos de geolocalización, por nombrar sólo algunos, se ha convertido en parte integral de nuestra vida social, trabajo y aprendizaje. Los dispositivos son cada vez más pequeños, económicos, portátiles e interconectados. Si bien es posible usar las nuevas tecnologías para recrear pedagogías tradicionales, centradas en la transmisión de conocimientos, los contextos de aprendizaje se han modificado constituyéndose en posibilitadores de nuevos aprendizajes. Como expresa Nicolás Burbulles, "las formas radicalmente nuevas en que las personas interactúan con la tecnología en el presente también proporcionan una valiosa oportunidad para que los docentes reformulen su trabajo y su función". [8]

Bill Cope y Mary Kalantzis [9] se refieren al aprendizaje ubicuo y lo definen en forma general como sigue: "el aprendizaje ubicuo representa un nuevo paradigma educativo que en buena parte es posible gracias a los nuevos medios digitales". La relación entre ubicuidad del aprendizaje y tecnologías no es biunívoca, se debe basar en la computación ubicua, pero requiere opciones metodológicas, tales como la colaboración, para hacer posible su aplicación práctica.

En síntesis, el aprendizaje colaborativo y ubicuo permite adquirir competencias para el trabajo en equipo y desarrollar habilidades requeridas en el ámbito profesional y laboral, habilidades deseables para los futuros ingenieros. Enriquecer puntos de vista desde perspectivas diferentes puede contribuir a una formación ciudadana basada en el respeto y la participación constructiva para la solución de problemas sociales de interés común. Citando nuevamente a Cope y Klantzis [9]: "Las tecnologías son el producto de las necesidades sociales. Cuando trabajan para nosotros, sus beneficios suelen ser más revolucionarios que sus especificaciones técnicas". 


\section{Contexto del trabajo}

La asignatura "Programación" forma parte del plan de estudios de la carrera Ingeniería Electrónica con orientación en Sistemas Digitales. Dicha asignatura, que se ubica en el segundo año de la Carrera, tiene como objetivo proporcionar al alumno herramientas que le permitan resolver problemas de cálculo numérico. Es la segunda asignatura con contenidos sobre programación que posee dicha carrera. Está estructurada en dos partes: la primera de ellas tiene como objetivo el aprendizaje del sistema operativo GNU/Linux: distribuciones, comandos, consola, interfaz gráfica y aplicaciones. La segunda parte tiene como objetivo profundizar el uso del lenguaje $\mathrm{C}$ para la resolución de problemas reales, trabajando con variables punteros, estructuras de datos y archivos. A partir de la formulación de problemas, los alumnos deben pensar soluciones de una forma creativa y expresarlas de una manera precisa, utilizando las herramientas adecuadas y buenas prácticas de programación.

La metodología utilizada por la cátedra consistía en clases teóricas de tipo expositivas, a cargo del profesor responsable, y posteriormente actividades de aula y laboratorio en forma individual, con la asistencia de un jefe de trabajos prácticos y auxiliares de docencia. Todas estas actividades se realizaban en forma presencial. El material de estudio podía descargarse del sitio web de la materia.

A partir del año 2008 el Centro de Informática Educativa (CIE) dependiente del área de Informática Educativa del Departamento de Informática pone a disposición de docentes y alumnos de la Universidad Nacional de San Luis (UNSL) el EVA "Aulas Virtuales" implementado sobre la plataforma Moodle. Con la creación del aula virtual de la materia, en el año 2013, surgen nuevas estrategias metodológicas pensadas para complementar la clase presencial. La siguiente tabla muestra la nueva diagramación de la materia (Tabla 1). Es oportuno señalar que, además de dejar todo el material disponible en el aula virtual, se proponen actividades para resolver en forma colaborativa, por ejemplo foros de preguntas y respuestas y/o foros generales, sobre cuestiones teóricas importantes.

\begin{tabular}{|c|c|c|c|c|c|c|c|c|c|c|c|}
\hline $\begin{array}{l}\text { Conte- } \\
\text { nidos }\end{array}$ & 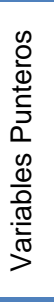 & 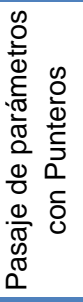 & $\sum_{\substack{\supset\\
}}$ & & 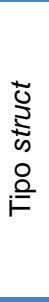 & 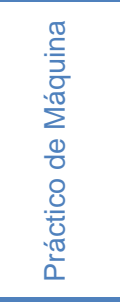 & 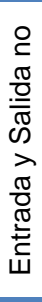 & 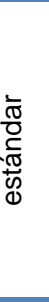 & 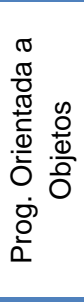 & 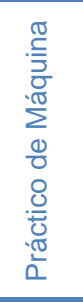 & 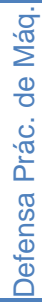 \\
\hline $\begin{array}{c}\text { Práctico } \\
\mathrm{N}^{\circ}\end{array}$ & 1 & 2 & 3 & 4 & 5 & Parte A & 6 & 7 & 8 & $\begin{array}{c}\text { Parte } \\
\text { B } \\
\end{array}$ & \\
\hline $\begin{array}{c}\text { Tipo de } \\
\text { Actividad }\end{array}$ & \multicolumn{5}{|c|}{ Individual } & Grupal & \multicolumn{3}{|c|}{ Individual } & \multicolumn{2}{|c|}{ Grupal } \\
\hline $\begin{array}{l}\text { Tipo de } \\
\text { Aula }\end{array}$ & \multicolumn{2}{|c|}{$\begin{array}{l}\text { Prácticas } \\
\text { en aula }\end{array}$} & \multicolumn{9}{|c|}{ Prácticas en Laboratorio de computadoras } \\
\hline
\end{tabular}

Tabla 1: Cronograma de la materia "Programación" 
El práctico de máquina debe resolverse en forma grupal, la colaboración se puede llevar adelante tanto en las clases presenciales, como en los foros dispuestos para tal fin. La incorporación en la asignatura de un aula virtual usando este EVA, posibilita un marco educativo más flexible y complementa la enseñanza presencial, permite al equipo de cátedra incorporar estrategias y desarrollar habilidades para el trabajo en grupo y el aprendizaje colaborativo. [10]

\section{Diagnóstico inicial}

Teniendo en cuenta la experiencia recuperada a partir del seguimiento realizado durante 3 años consecutivos de dictado de la asignatura (período 2011-2013), se detectó un alto porcentaje de deserción y un bajo rendimiento académico de los alumnos.

Este seguimiento fue realizado por los docentes de la cátedra considerando resultados de parciales, asistencia a clases prácticas y teóricas, corrección de trabajos prácticos, muestra y resolución de parciales. Indica falta de hábitos de estudio, falencias en la comprensión de textos (puesta en evidencia en la dificultad para la resolución de problemas) e inconvenientes para cumplir con los horarios establecidos.

\section{Estrategias metodológicas}

A partir de la incorporación de herramientas de SL para facilitar el aprendizaje ubicuo fue necesario realizar modificaciones en la metodología utilizada. Se decidió utilizar un entorno integrado de desarrollo (IDE) denominado CODE::BLOCKS [11]. Esta herramienta permitió trabajar en un ambiente con una interfaz común para poder editar, compilar, depurar y ejecutar códigos en lenguaje $\mathrm{C}$, facilitando la tarea de programación.

Si bien esta herramienta no funciona en forma integrada con el aula virtual, la propuesta didáctica para el práctico grupal de máquina se llevó a cabo a través de un foro de discusión creado dentro del EVA.

Utilizando las actividades y recursos disponibles en el aula virtual, se desarrollaron como complemento a los trabajos prácticos de aula y las evaluaciones parciales: lectura de materiales de apoyo desarrollado por la cátedra, discusión en foros, actividades colaborativas para resolver ejercicios propuestos, visualización de videos tutoriales, cuestionarios y entrega de tareas planificadas (códigos).

Es importante mencionar que con el objetivo de desarrollar habilidades de colaboración y favorecer el compromiso con el propio proceso de aprendizaje, se propone el práctico de máquina en forma grupal. Los estudiantes eligen libremente los grupos de trabajo, conformando equipos de dos integrantes. Cada grupo cuenta con la guía de un tutor que promueve la colaboración y el intercambio de conocimiento entre pares. El práctico de máquina consiste en resolver un problema real e implementar una solución en lenguaje $\mathrm{C}$, utilizando el IDE CODE:BLOCKS bajo el sistema operativo GNU/Linux. 


\section{Recolección de datos}

Con el propósito de analizar resultados y modificar las prácticas de enseñanza, se obtuvo información a través de diferentes instrumentos: el Diagnostico Inicial explicado en el punto 4, la información de seguimiento provista por el EVA y la encuesta de satisfacción, diseñada por la cátedra, para conocer la opinión de los alumnos.

La encuesta de satisfacción consta de 11 preguntas sobre los siguientes aspectos: desempeño docente, estrategias y materiales elaborados por la cátedra y un espacio especial para que el alumno especifique sugerencias y/o comentarios. (Anexo I).

El cuestionario elaborado se aplicó a todos los alumnos matriculados en la asignatura, en los horarios y aulas habituales que utiliza la cátedra. Una vez completado en forma anónima se procedió a la interpretación de la información para obtener conclusiones.

La Figura 1 refleja que únicamente un 14\% de los alumnos asisten a las clases presenciales según lo recomendado por la cátedra y un porcentaje mayor al $80 \%$ no puede asistir. Esto confirma la necesidad de proponer estrategias alternativas.

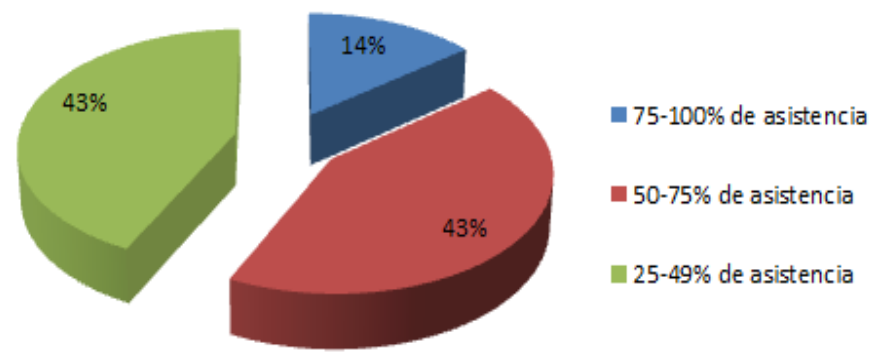

Figura 1: Asistencia a las clases presenciales

La Figura 2 refleja que la mitad de los alumnos tienen inconvenientes de asistencia por cuestiones laborales. Por otra parte, un $22 \%$ de los alumnos presentan incompatibilidad horaria debido a que cursan materias de diferentes años de su plan de estudio.

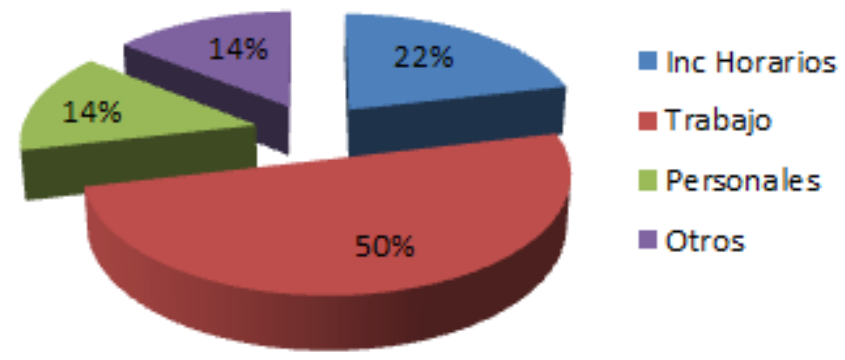

Figura 2: Motivos de inasistencias 
La Figura 3 indica que la mayoría de los alumnos consideran adecuado el uso de la plataforma "Aulas virtuales" como complemento a las actividades presenciales.

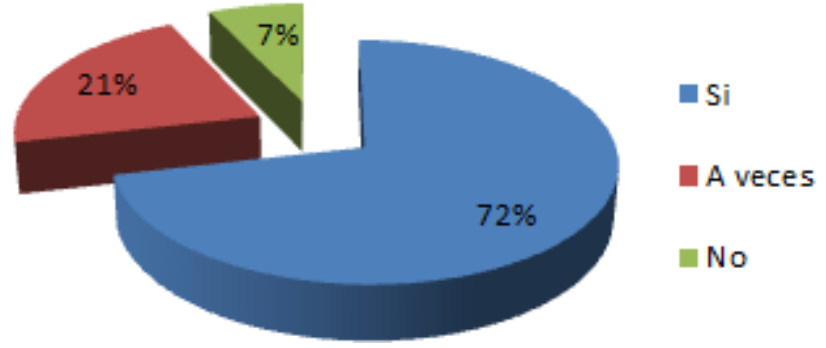

Figura 3: Utilización de "Aulas virtuales"

La Figura 4 señala que únicamente el 7\% de los alumnos encuentra dificultades con la metodología utilizada por la cátedra para la realización de esta tarea.

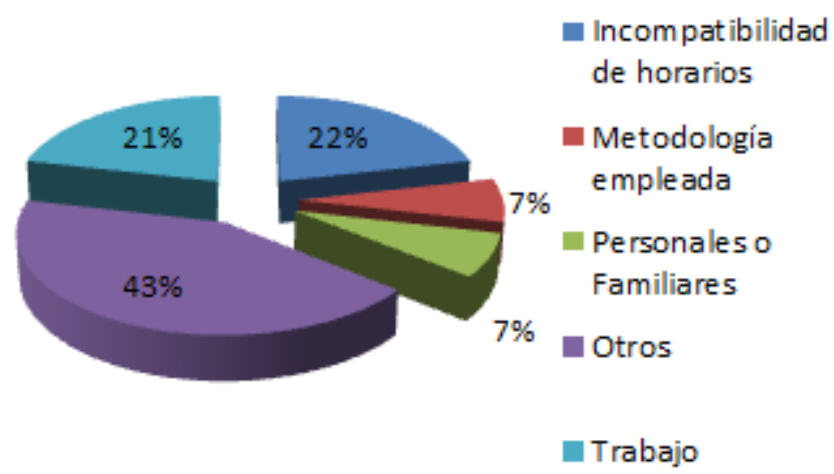

Figura 4: Problemas en la realización del práctico de máquina

La Figura 5 indica que los alumnos consideran adecuadas las estrategias y recursos utilizados por la cátedra.
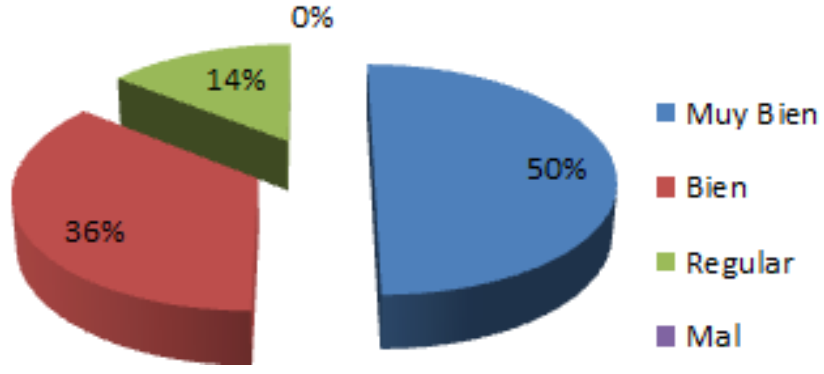

Figura 5: Uso de métodos y recursos didácticos adecuados

A los resultados anteriores se suman los datos del rendimiento académico del período 2011-2014 para cotejarlos. 
La Figura 6 muestra una comparativa de resultados académicos para las condiciones: Libre por Faltas, Libre por Parcial, Regular y Promoción.

En cuanto a la condición de Libre por Faltas: si bien en el año 2012 existe una disminución importante, en el año 2013 aumenta nuevamente, pero en el año 2014 se registran los porcentajes más bajos de deserción del período analizado.

Se puede observar una disminución progresiva en porcentaje de los alumnos Libres por Parcial y un aumento significativo de aquellos que aprueban la materia, Regular y Promoción.

\section{Rendimiento Académico}

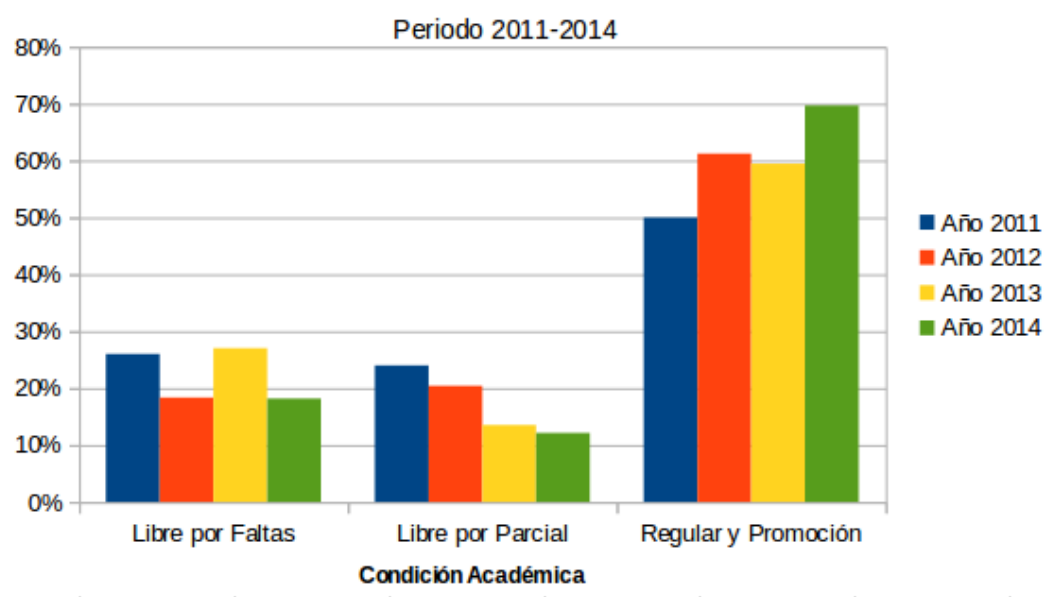

Figura 6: Análisis comparativo períodos 2011-2014

\section{7- Conclusiones}

A partir de 2014 se fortaleció el uso del aula virtual en el desarrollo de actividades colaborativas, lo que impactó positivamente en el rendimiento académico. Desde el punto de vista del docente, el uso de este modelo implicó modificar significativamente el rol, en lo que respecta a la elaboración de materiales pedagógicos, creación y mantenimiento del aula virtual, seguimiento del proceso de aprendizaje y comunicación con los alumnos. Por otra parte, posibilitó el seguimiento continuo de los aprendizajes mediante la lectura de los foros y los informes estadísticos provistos por el EVA, para ofrecer la ayuda necesaria en el momento oportuno. El uso del EVA “Aulas virtuales” y el IDE CODE::BLOCKS permitió a los alumnos trabajar colaborativamente, con independencia de horarios y espacio. Ofreció vías de comunicación más ágiles y contribuye a una formación más flexible durante el proceso de enseñanza y aprendizaje, mediante la realización de actividades no presenciales. Además, brindó respuesta a las demandas expresadas en la encuesta de satisfacción en cuanto a las actividades grupales. 
En síntesis, los resultados académicos obtenidos mediante el uso del EVA “Aulas Virtuales" como complemento a la clase presencial y el IDE CODE::BLOCKS, avalan la continuidad de esta línea de trabajo. Surge como alternativa evaluar la posibilidad de utilizar un módulo para programación integrado en el EVA. Esto permitiría realizar un mejor seguimiento del estado de resolución del práctico de máquina, aprovechando los beneficios de usar una interfaz única.

\section{Referencias}

1 Sitio oficial de Moodle. Documentación en línea. Disponible en: www.moodle.org

2 Aguirre Jesús, Viano Hugo, García Berta: Entornos virtuales y herramientas de software libre como apoyo a la enseñanza de programación. Modalidad Poster. $2^{\circ}$ Congreso Nacional de Ingeniería Informática-CONAIISI. San Luis. (2014).

3 Viano, Hugo \& Aguirrre, Jesús: Uso de aulas virtuales como apoyo a la enseñanza de Programación. En: Chiarani, Daza y Allende (Coords). Cultura digital en la Universidad Nacional de San Luis. pp. 51 a 53 Nueva Editorial Universitaria. ISBN: 978-987-1852-97-0 (2014)

4 Coll, C. \& Monereo, C. Educación y aprendizaje en el siglo XXI: Nuevas herramientas, nuevos escenarios, nuevas finalidades. En C. Coll y C. Monereo (Eds.) Psicología de la Educación Virtual (pp.19-53). Madrid: Ediciones Morata. (2008)

5 Johnson, D.W. Johnson, R.T.,\& Holubec, E.J.: El aprendizaje cooperativo en el aula. Barcelona: Paidós. (1999).

6 Guitert, M.; Giménez, F.: El trabajo cooperativo en entornos virtuales de aprendizaje. En: Duart, J.M.; Sangra, A. (Ed.) Aprender en la virtualidad, pp. 113 -134. Barcelona: Gedisa. (2000)

7 Gunawardena, Ch., Lowe, C. \& Anderson, T.: Analysis of a global online debate and the development of an interaction analysis model for examining social construction of knowledge in computer conferencing. J. Educational Computing Research, vol. 17, núm. 4, pp. 395-429. (1997).

8 Nicholas C. Burbules: El aprendizaje ubicuo y el futuro de la enseñanza, Encounters/Encuentros/Rencontres on Education, Vol. 13, 2012, pág 3 a 14. (2012)

9 Cope Bill y Kalantzis, M.- Aprendizaje ubicuo - Grupo nodos ELE. (2009)

10 Mondéjar, J., Mondéjar, J. A. \& Vargas, M.: Implantación de la metodología e- learning en la docencia universitaria: una experiencia a través del proyecto Campus Virtual. Revista Latinoamericana de Tecnología Educativa, 5 (1), 59-71. (2006). [http://www.unex.es/didactica/RELATEC/sumario_5_1.htm]

11 CODE::BLOCKS Sitio oficial: http://www.codeblocks.org/

12 Area, M.y Adell, J.: eLearning: Enseñar y aprender en espacios virtuales. En J. De Pablos (Coord): Tecnología Educativa. La formación del profesorado en la era de Internet. Aljibe, Málaga, pags. 391-424. (2009) 


\section{Anexo I: Encuesta de satisfacción a Estudiantes - 2 Cuat. de 2014}

1- ¿Cuántas veces te has matriculado en esta asignatura?
A. 1
B. 2
C. 3 o más

2- ¿Cuál ha sido tu asistencia a las clases de esta asignatura?
A. $0-25 \%$
B. $25-49 \%$
C. $50-75 \%$
D. $75 \%-100 \%$

3- ¿Cuál es el grado de dificultad de esta asignatura con respecto a otras de la titulación?
A. Muy fácil
B. Fácil
C. Normal
D. Difícil
E. Muy Difícil

4- ¿Cuáles son los motivos de las faltas de asistencia?
A. Trabajo
B. Incompatibilidad de horarios
C. Personales
D. Metodologia empleada
E. Otros

5- ¿Cómo considera que fue su participación en los foros de la plataforma "Aulas Virtuales" durante la cursada?
A. Muy Pobre
B. Pobre
C. Activa
D. Muy Activa

6- Considera que el uso de la plataforma "Aula Virtual" mejoró sus posibilidades de aprender?
A. $\mathrm{Si}$
B. No
C. A veces

7- Cuáles son los principales problemas en la realización del trabajo práctico de máquina:
A. Trabajo
B. Incompatibilidad de horarios
C. Personales
D. Metodologia empleada
E. Otros

8- Considera necesario incluir otras herramientas para favorecer el aprendizaje de la asignatura como:

A. Redes sociales: Facebook, Twitter, etc.

B. La nube: Dropbox, Skydrive, etc.

C. Otros

9- La Cátedra usa métodos y recursos didácticos adecuados para favorecer el aprendizaje de la asignatura (pizarra, transparencias, recursos audiovisuales, etc):
A. Regular
B. Bien
C. Muy Bien

10- La actividad grupal del trabajo práctico de máquina de la asignatura es:
A. Muy fácil
B. Fácil
C. Normal
D. Difícil
E. Muy difícil

11- ¿Cuáles son las actividades que considera necesaria incorporar a la asignatura?

Cuestionarios
A. Encuestas
B. Trabajos grupales
C. Ejemplos
D. Otros 